\title{
3 Research Square

\section{A nomogram to predict the risk of low cardiac output syndrome after heart valve replacement in cardiac valvular disease patients}

\section{Qiusha Qing}

people's hospital of deyangcity

\section{Xin Wei}

Peoples Hospital of Deyang City

Hong Zhen

Peoples Hospital of Deyang City

\section{Zhi Wen}

Peoples Hospital of Deyang City

\section{Xiaokang Sun}

Peoples Hospital of Deyang City

Junrong Yang

Peoples Hospital of Deyang City

\section{Peirui Chen ( $\nabla 01706394 @ q q . c o m$ )}

Peoples Hospital of Deyang City https://orcid.org/0000-0001-9284-9543

\section{Research article}

Keywords: nomogram, low cardiac output syndrome, heart valve replacement

Posted Date: January 14th, 2021

DOl: https://doi.org/10.21203/rs.3.rs-143778/v1

License: (c) (i) This work is licensed under a Creative Commons Attribution 4.0 International License. Read Full License 


\section{Abstract}

Background and Aim

Low cardiac output syndrome (LCOS) is a serious postoperative complication, affecting the prognosis of patients underwent heart valve replacement (HVR). We aim to create a nomogram to predict LCOS after HVR in cardiac valvular disease patients.

\section{Methods}

We performed a retrospective review of 500 cardiac valvular disease patients underwent HVR from 2016 to 2020 in our department. Univariate analysis evaluated the associations between clinical/echocardiographic parameters and LCOS. Independent t-test or Mann-Whitney U-test: for continuous variables. Fisher's exact test or $\chi 2$ test: for categorical variables. Variables with a $P<0.1$ in the univariate analysis were entered into least absolute shrinkage and selection operator (LASSO) regression to select factors. Then, multivariable logistic regression was performed to develop the predictive model and a nomogram. The discrimination ability, calibration curve analysis and decision curve analysis (DCA) of the nomogram was evaluated in development and validation group.

Results

Of 500 patients, 92 developed postoperative LCOS (18.4\%). The nomogram included the following variables: body mass index (BMI), left ventricular ejection fraction (LVEF) and global longitudinal strain (GLS). The nomogram showed favorable calibration and favorite performance for LCOS detection with Cindex 0.826 in the development group and 0.783 in validation group. The DCA showed that the novel model was clinically useful.

\section{Conclusions}

We created a nomogram of predicting postoperative LCOS in cardiac valvular disease patients received HVR. This nomogram could be an important tool of LCOS risk prediction after HVR to guide the therapeutic strategy in cardiac valvular disease patients.

\section{Introduction}

Cardiac valvular disease is a commonly seen heart disorder, accounting for one-fourth of all in-hospital admissions for cardiovascular disease in developed countries [1]. Although an increasing number of patient subgroups are being considered for transcathether valve replacement, transcatheter mitral valve replacement (TMVR) or transcathether aortic valve replacement (TAVR), surgical heart valve replacement (HVR) remains the mainstay treatment [2]. With the maturity of cardiac surgical technique in china, the surgery-associated mortality rate has dropped considerably in the past few years [3]. However, the rate of major cardiovascular complications remains high $[1,4]$. Low cardiac output syndrome (LCOS) is the most serious complication, which associated with increased morbidity, and treatment-related costs [5]. Recent 
studies suggest a wide LCOS incidence of 3.9-41\% [3, 6, 7]after heart valve surgery. For these patients, LCOS is associated with a 30-day mortality rate of $12.8-38 \%[3,6,8]$. So, early identification and risk assessment of LCOS in patients undergoing HVR are important to improving the treatment and prognosis of these patients.

Nowadays, several factors have been proposed associated with LCOS after HVR, including advanced age, malnutrition, impaired left ventricular (LV) function, global longitudinal strain (GLS), New York Heart Association class IV, renal failure, earlier year of operation, female gender and shock before surgery [4]. Strain-imaging by speckle-tracking echocardiography is a technique to directly quantify the extent of myocardial contractility and has better prognostic value than ejection fraction [9]. As GLS is most reproducible and commonly used strain parameter, it has recommended to be the parameter used to describe LV systolic function in patients with valvular heart disease and cardiomyopathies [10-13]. But it has not been widely accepted and not related to the visual prediction model of LCOS in patients underwent HVR.

A nomogram is a graphic score based on a statistical predictive model for determining the probability of clinical outcomes in an individual patient [14]. To date, nomograms for predicting the risk of LCOS in cardiac valvular disease patients treated by HVR have not been reported. In this study, we aimed to develop a nomogram for predicting the risk of LCOS for patient after HVR.

\section{Materials And Methods}

\section{Patient selection}

We retrospectively included 318 patients who underwent heart valve replacement (HVR) with cardiopulmonary bypass (CPB) in our institution from January 2016 to December 2018 in the development group, including 60 patients with LCOS and 258 patients without LCOS. A total of 182 patients underwent HVR from January 2019 to October 2020 were enrolled in the validation group, including 32 patients with LCOS and 150 patients without LCOS. The clinical data were collected from the data system of our hospital, the ultrasound images were collected from the department of ultrasonography of People's Hospital of DeyangCity.

The inclusion criteria were (1) cardiac valvular lesion was confirmed by preoperative echocardiography; (2) complete clinical, laboratory and imaging data available. The exclusion criteria were (1) patients aged < 18 years; (2) previous cardiac surgery history; (3) emergency operation; (4) with serious coronary heart disease or acute decompensated heart failure (ADHF); (5) more than moderately depressed left ventricular ejection fraction (LVEF) $(<40 \%)$. The study was approved by ethics committee of People's Hospital of DeyangCity, and informed consent was obtained from all individuals.

\section{Outcomes}


LCOS was our primary outcome which defined as: A systolic blood pressure $<90 \mathrm{~mm} \mathrm{Hg}$ for at least 30 minutes after correcting hypovolemia or requirement of inotropic medications (dopamine, dobutamine, milnirone or norepinephrine) or an intra-aortic balloon pump (IABP) for at least $12 \mathrm{~h}$ to maintain systolic blood pressure $>90 \mathrm{~mm} \mathrm{Hg}$ and the cardiac output $>2.2 \mathrm{~L} / \mathrm{min} / \mathrm{m} 2$. And presence of at least one of the following, pulmonary capillary wedge pressure (PCWP) $>18 \mathrm{mmHg}$, difference between peripheral skin temperature and core body temperature $>5^{\circ} \mathrm{C}$, central venous oxygen saturation $<60 \%$, urinary output less than $0.5 \mathrm{ml} / \mathrm{kg} / \mathrm{h}$. 30 day mortality, ICU and hospital stay were our secondary outcome.

\section{Risk factors}

We collected and analyzed the following factors of the subjects: general information (gender, age, body mass index (BMI), New York Heart Association (HYHA ) class, diabetes, hypertension, congestive heart failure (CHF), atrial fibrillation (AF), serum creatinine(SCR), and blood urea nitrogen (BUN) ); echocardiographic parameters (heart valve lesion type, LVEF, left ventricular end diastolic diameter (LVEDD), left ventricular end systolic diameter (LVESD), interventricular septum thickness (IVST), left ventricular posterior wall thickness(LVPWT), left atrial diameter(LAD), GLS ); intraoperative and postoperative parameters (types of prosthetic valve, cardiopulmonary bypass time (CPBT), aortic crossclamp time (ACCT), blood loss, transfusion, post-surgical AF, infection, and post-surgical acute renal failure).

Preoperative transthoracic echocardiogram (TTE) was performed by using a Philips EPIQ 7C (Philips Medical Systems, Andover, MA) with a S5-1 probe (frequency ratio $1.0 \sim 5.0 \mathrm{MHz}$ ). The LVEDD, LVESD, LAD, IVST and LVPWT were measured by two-dimensional ultrasound on the long axis of the left ventricle. The left ventricular ejection fraction (LVEF) was measured by Simpson's biplane method. Twodimensional dynamic image of two-chamber, three-chamber and apical four chamber view were collected with a frame rate ranging from $60 \sim 80$ frames/s. The original data was stored in mobile hard disk with DICOM format for off-line analysis. The images were processed and analyzed by QLab 10.0 software (Phillips Medical Systems), and the region of interest was automatically mapped by the system. Manual adjustments of the region of interest were made whenever necessary to optimize myocardial tracking. Then automated cardiac motion quantification (aCMQ) technology was applied to calculate GLS of the left ventricular systolic period.

\section{Statistical analysis}

For normally distributed continuous variables, a Student's t-test was performed, and for non-normally distributed continuous variables non-parametric Mann-Whitney $\mathrm{U}$ test was used. For the categorical variables, the Chi-squared test and Fisher's exact test was used. Two-sided $p$-values $<0.05$ was considered as statistical significance. As the low incidence of primary outcome in our study, the least absolute shrinkage and selection operator (LASSO) [15] method was used to select the best predictive characteristics of risk factors from HVR patients with LCOS. Variables showing $P<0.1$ in the univariate analysis were included in the LASSO regression model. Nonzero features were selected in the LASSO regression model. Then, the selected predictive factors were used to formulate a nomogram to estimate 
the risk of LCOS in HVR patients by the multivariate logistic regression analysis. The predictive accuracy of the constructed nomogram was estimated using index of probability of concordance (C-index) and a visualized receiver operator characteristic (ROC) curve. The relative corrected $\mathrm{C}$-index was calculated by boot validation (1000 boot resamples). Calibration was plotted to explore the predictive accuracy of the nomogram by bootstrapping with 1000 resamples. To evaluate the added clinical utility of nomogram in predicting LCOS for HVR patients, decision curve analysis (DCA) was performed by quantifying the net benefits for a range of threshold probabilities in the combined development and validation dataset. All statistical analyses were performed and graphs were constructed using IBM SPSS Statistics (Version 23.0; IBM Corp., New York, USA) and R software (Version 4.0.3; R Foundation for Statistical Computing, Vienna, Austria).

\section{Results}

\section{All patients' clinical and echocardiographic characteristics}

We recruited 562 patients from January 2016 to October 2020 at our center, 73 patients were excluded: 7 cardiac surgery history, 11 ADHF, 10 serious coronary heart disease, $20 \mathrm{LVEF}<40 \%$, and 25 echocardiographic views cannot be used to measure GLS. Finally, 318 patients in the development group and 182 patients in the validation group were included (Fig. 1). In development group, 62 patients had LCOS (18.8\%). There were significant differences in age, BMI, HYHA class, CHF, LVEF, and GLS between the LCOS and no- LCOS patients $(P<0.05)$. In the verification group, 32 patients had LCOS $(17.6 \%)$. There were significant differences in age, BMI, HYHA class, CHF, valve replacement, CBPT, ACCT, LVEF, and GLS between the LCOS and no- LCOS patients $(P<0.05)$. Detailed information on the univariate analysis of clinical and echocardiographic characteristics of the patients is shown in Table 1 and Table 2. 30 day mortality occurred more frequently and the mean hospital stay time and ICU stay time were longer among patients who developed LCOS $(P<0.05)$ (Table 3$)$.

Table 1 Univariate analysis of clinical characteristics in patients with and without LCOS 


\begin{tabular}{|c|c|c|c|c|c|c|}
\hline \multirow[t]{3}{*}{ Variable } & \multicolumn{2}{|c|}{$\begin{array}{l}\text { Development group } \\
(\mathrm{n}=318)\end{array}$} & \multirow[t]{3}{*}{$\begin{array}{l}P \\
\text { value }\end{array}$} & \multicolumn{2}{|c|}{$\begin{array}{l}\text { Validation group } \\
(\mathrm{n}=182)\end{array}$} & \multirow[t]{3}{*}{$\begin{array}{l}P \\
\text { value }\end{array}$} \\
\hline & Non- & LCOS & & Non-LCOS & LCOS & \\
\hline & $(\mathrm{n}=258)$ & $(n=60)$ & & $(n=150)$ & $(n=32)$ & \\
\hline Age, years, n (\%) & & & $<0.001$ & & & 0.002 \\
\hline$<60$ & $113(43.8)$ & $15(25.0)$ & & $69(46.0)$ & $4(12.5)$ & \\
\hline $60-70$ & $76(29.5)$ & $36(60.0)$ & & $43(28.7)$ & $14(43.8)$ & \\
\hline$>70$ & $69(26.7)$ & $9(15.0)$ & & $38(25.3)$ & $14(43.8)$ & \\
\hline Gender, n (\%) & & & 0.978 & & & 0.674 \\
\hline Male & $107(41.5)$ & $25(41.7)$ & & $55(36.7)$ & $13(40.6)$ & \\
\hline Female & $151(58.5)$ & $35(58.3)$ & & $95(63.3)$ & 19(59.4) & \\
\hline BMI, n (\%) & & & 0.007 & & & 0.019 \\
\hline $18.5-27.9$ & 199(77.1) & $46(76.7)$ & & $109(72.7)$ & 19(59.4) & \\
\hline$\geqq 28$ & $49(19.0)$ & $6(10.0)$ & & $36(24.0)$ & $8(25.0)$ & \\
\hline$<18.5$ & $10(3.9)$ & $8(13.3)$ & & $5(3.3)$ & $5(15.6)$ & \\
\hline HYHA class, n (\%) & & & 0.022 & & & 0.003 \\
\hline$\nabla$ & $84(32.6)$ & 13(21.7) & & $46(30.7)$ & $3(9.4)$ & \\
\hline Q & $137(53.1)$ & $30(50.0)$ & & $87(58.0)$ & 19(59.3) & \\
\hline III & $37(14.3)$ & $17(28.3)$ & & $17(11.3)$ & 10(31.3) & \\
\hline Diabetes, n (\%) & & & 0.136 & & & 0.618 \\
\hline NO & $222(86.0)$ & 47(78.3) & & $126(84.0)$ & $28(87.5)$ & \\
\hline YES & $36(14.0)$ & 13(21.7) & & $24(16.0)$ & $4(12.5)$ & \\
\hline Hypertension, n (\%) & & & 0.772 & & & 0.250 \\
\hline NO & $180(69.8)$ & 43(71.7) & & $109(72.7)$ & $20(62.5)$ & \\
\hline YES & 78(31.2) & 17(28.3) & & $41(27.3)$ & $12(37.5)$ & \\
\hline $\mathrm{AF}, \mathrm{n}(\%)$ & & & 0.091 & & & 0.097 \\
\hline NO & $231(89.5)$ & 49(81.7) & & $130(86.7)$ & $24(75.0)$ & \\
\hline
\end{tabular}




\begin{tabular}{|c|c|c|c|c|c|c|}
\hline YES & $27(10.5)$ & $11(18.3)$ & & $20(13.3)$ & $8(25.0)$ & \\
\hline CHF, n (\%) & & & 0.028 & & & 0.066 \\
\hline NO & $223(86.4)$ & $45(75.0)$ & & $128(85.3)$ & 23(71.9) & \\
\hline YES & $35(13.6)$ & $15(25.0)$ & & $22(14.7)$ & $9(28.1)$ & \\
\hline SCR, mmol/L (IQR) & $\begin{array}{l}77.08 \\
(65.63- \\
83.82)\end{array}$ & $\begin{array}{l}78.85 \\
(71.72- \\
88.18)\end{array}$ & 0.089 & $\begin{array}{l}76.55(64.90- \\
82.84)\end{array}$ & $\begin{array}{l}75.01 \\
(66.06- \\
86.28)\end{array}$ & 0.776 \\
\hline $\begin{array}{l}\mathrm{BUN}, \mathrm{mmol} / \mathrm{L} \\
\text { (IQR) }\end{array}$ & $\begin{array}{l}6.72 \\
(4.89- \\
8.50)\end{array}$ & $\begin{array}{l}7.02 \\
(5.88- \\
8.60)\end{array}$ & 0.066 & $\begin{array}{l}6.34 \\
(4.9-8.0)\end{array}$ & $\begin{array}{l}6.18 \\
(5.28- \\
7.89)\end{array}$ & 0.919 \\
\hline $\begin{array}{l}\text { Intraoperative and } \\
\text { Postoperative }\end{array}$ & & & & & & \\
\hline Valve type, n (\%) & & & 0.462 & & & 0.351 \\
\hline $\begin{array}{l}\text { Bioprosthetic } \\
\text { Valve }\end{array}$ & 86(33.3) & $23(38.3)$ & & $57(38.0)$ & $15(46.9)$ & \\
\hline $\begin{array}{l}\text { Mechanical } \\
\text { Valve }\end{array}$ & $172(66.7)$ & $37(61.7)$ & & $93(62.0)$ & $17(53.1)$ & \\
\hline $\begin{array}{l}\text { Valve replacement, } n \\
\text { (\%) }\end{array}$ & & & 0.144 & & & 0.02 \\
\hline AV & $100(38.8)$ & $30(50.0)$ & & $90(60.0)$ & $11(34.4)$ & \\
\hline MV & $131(50.8)$ & $22(36.7)$ & & $35(23.3)$ & $11(34.4)$ & \\
\hline $\mathrm{AV}+\mathrm{MV}$ & $27(10.5)$ & $8(13.3)$ & & $24(16.7)$ & $10(31.2)$ & \\
\hline Bloodloss, ml, n (\%) & & & 0.927 & & & 0.303 \\
\hline$<600$ & $212(82.2)$ & $49(81.7)$ & & 116(77.3) & $22(68.8)$ & \\
\hline$\geqq 600$ & $46(17.8)$ & 11(18.3) & & $34(22.7)$ & 10(31.2) & \\
\hline Transfusion, n (\%) & & & $0.705^{\star}$ & & & $0.692 *$ \\
\hline NO & $9(3.5)$ & $3(5.0)$ & & $10(6.7)$ & $1(3.3)$ & \\
\hline YES & $249(96.5)$ & $57(95.0)$ & & $140(93.3)$ & $31(96.7)$ & \\
\hline CBPT, min, n (\%) & & & 0.682 & & & 0.029 \\
\hline$\leqq 78$ & $90(34.9)$ & $20(33.3)$ & & $67(44.7)$ & $7(21.9)$ & \\
\hline
\end{tabular}




\begin{tabular}{|c|c|c|c|c|c|c|}
\hline 79- 91 & $80(31.0)$ & $22(36.7)$ & & $40(26.7)$ & $9(28.1)$ & \\
\hline$\geqq 92$ & $88(34.1)$ & $18(30.0)$ & & $43(28.7)$ & $16(50.0)$ & \\
\hline ACCT, min, $n(\%)$ & & & 0.323 & & & 0.017 \\
\hline$\leqq 47$ & $90(34.9)$ & $20(33.3)$ & & $70(46.7)$ & $8(25.0)$ & \\
\hline $48-60$ & $79(30.6)$ & $24(40.0)$ & & $38(25.3)$ & $7(21.9)$ & \\
\hline$\geqq 61$ & $89(34.5)$ & $16(26.7)$ & & $42(28.0)$ & $17(53.1)$ & \\
\hline Postoperation AF, n (\%) & & & 0.589 & & & 0.106 \\
\hline NO & $214(82.9)$ & $48(80.0)$ & & $126(84.0)$ & 23(71.9) & \\
\hline YES & $44(17.1)$ & $12(20.0)$ & & $24(16.0)$ & $9(28.1)$ & \\
\hline $\begin{array}{l}\text { Postoperation delirium, } \\
\mathrm{n}(\%)\end{array}$ & & & $1.000 *$ & & & $0.148 *$ \\
\hline NO & 242(93.8) & $56(93.3)$ & & $145(96.7)$ & $29(90.6)$ & \\
\hline YES & $16(6.2)$ & $4(6.7)$ & & $5(3.3)$ & $3(9.4)$ & \\
\hline Infection, n (\%) & & & 0.312 & & & 0.364 \\
\hline NO & $220(85.3)$ & $38(63.3)$ & & $131(87.3)$ & $26(81.3)$ & \\
\hline YES & $38(14.7)$ & $22(36.7)$ & & 19(12.7) & $6(18.7)$ & \\
\hline Renal failure, n (\%) & & & 0.099 & & & $1.000 *$ \\
\hline NO & $249(96.5)$ & $55(91.7)$ & & 142(94.7) & $31(96.9)$ & \\
\hline YES & $9(3.5)$ & $5(8.3)$ & & $8(5.3)$ & 1(3.1) & \\
\hline
\end{tabular}

BMI body mass index; NYHA New York Heart Association; AF atrial fibrillation; CHF congestive heart failure; SCR serum creatinine; BUN blood urea nitrogen; AV aortic valve; MV mitral valve; CBPT cardiopulmonary bypass time; ACCT aortic cross-clamp time

*Fisher's exact test

Table 2 Univariate analysis of echocardiographic parameters in patients with and without LCOS 


\begin{tabular}{|c|c|c|c|c|c|c|}
\hline \multirow[t]{3}{*}{ Variable } & \multicolumn{2}{|c|}{$\begin{array}{l}\text { Development group } \\
(\mathrm{n}=318)\end{array}$} & \multirow[t]{3}{*}{$P$ value } & \multicolumn{2}{|c|}{$\begin{array}{l}\text { Validation group } \\
(\mathrm{n}=182)\end{array}$} & \multirow[t]{3}{*}{$P$ value } \\
\hline & Non-LCOS & LCOS & & Non-LCOS & LCOS & \\
\hline & $(n=258)$ & $(n=60)$ & & $(n=150)$ & $(n=32)$ & \\
\hline LVEDD, mm & $\begin{array}{l}51.77 \pm \\
6.06\end{array}$ & $\begin{array}{l}52.80 \pm \\
5.36\end{array}$ & 0.227 & $\begin{array}{l}51.55 \pm \\
6.43\end{array}$ & $\begin{array}{l}52.66 \pm \\
7.05\end{array}$ & 0.388 \\
\hline LVESD, mm & $33.8 \pm 4.81$ & $34.08 \pm 5.08$ & 0.691 & $\begin{array}{l}34.43 \pm \\
5.53\end{array}$ & $34.63 \pm 5.79$ & 0.855 \\
\hline IVST, mm (IQR) & $9(8-9)$ & $9(9-10)$ & 0.154 & $9(8-10)$ & $9(8-10)$ & 0.128 \\
\hline $\begin{array}{l}\text { LVPWT, mm } \\
\text { (IQR) }\end{array}$ & $9(8-9)$ & $9(8-9)$ & 0.280 & $9(7-10)$ & $9(8-10)$ & 0.188 \\
\hline LAD, mm (IQR) & 39 (35-42) & $40(36-43)$ & 0.384 & $39(36-42)$ & $41(33-45)$ & 0.268 \\
\hline LVEF, \%, n (\%) & & & 0.002 & & & $<0.001$ \\
\hline$<50$ & $26(10.1)$ & $14(23.3)$ & & $11(7.3)$ & $9(28.1)$ & \\
\hline $50-60$ & $100(38.8)$ & $35(58.3)$ & & $67(44.7)$ & 19(59.4) & \\
\hline$>60$ & $132(51.2)$ & $11(18.3)$ & & $72(48)$ & $4(12.5)$ & \\
\hline GLS, n (\%) & & & $<0.001$ & & & 0.012 \\
\hline$\leqq-21$ & $99(38.4)$ & $1(1.7)$ & & $48(32)$ & $2(6.3)$ & \\
\hline$-20--17$ & 101(39.1) & $31(51.7)$ & & $62(41.3)$ & $16(50.0)$ & \\
\hline$\geqq-16$ & $58(22.5)$ & $28(46.7)$ & & $40(26.7)$ & $14(43.8)$ & \\
\hline
\end{tabular}

LVEF left ventricular ejection fraction; GLS global longitudinal strain; LVEDD left ventricular end diastolic diameter; LVESD left ventricular end systolic diameter; IVST interventricular septum thickness; LVPWT left ventricular posterior wall thickness; LAD left atrial diameter

Table 3 Outcomes of patients with and without LCOS 


\begin{tabular}{|llll|}
\hline Variable & \multicolumn{2}{c|}{ Cohort $(\mathrm{n}=500)$} & P value \\
\cline { 2 - 4 } & $\begin{array}{l}\text { Non-LCOS } \\
(\mathrm{n}=408)\end{array}$ & \multicolumn{1}{c|}{$(\mathrm{n}=92)$} & \\
& & & \\
\hline 30 day mortality, $\mathrm{n}(\%)$ & $5(1.2)$ & $14(15.2)$ & $<0.001$ \\
\hline Hospital stay, days (IQR) & $24(22-28)$ & $29(26-33.75)$ & $<0.001$ \\
\hline ICU stay length, days (IQR) & $2(2-4)$ & $4(3-5.75)$ & $<0.001$ \\
\hline
\end{tabular}

\section{Factor selection and Nomogram development}

Ten clinical and echocardiographic data with $P<0.1$ in the univariate analysis were included in LASSO regression analyses. Eventually, three potential predictors, BMI, LVFE, and GLS, on the basis of 318 patients in the development group were identified as predictors (Fig. 2). The results of the multivariate logistic regression analysis including these three predictors are given in Table 4. We then establish an individualized nomogram prediction model of LCOS based on these three selected variables (Fig. 3). The nomogram is used by scoring the points corresponding to each variable. The sum of scores for all variables is recorded as the total score, and the predicted risk corresponding to the total score is the probability of LCOS after HVR.

Table 4 Multivariate logistic regression analysis of prediction factors for LCOS

\begin{tabular}{|lllll|}
\hline Variable & $\beta$ & OR (odds ratio) & $95 \% \mathrm{Cl}$ & $P$ value \\
\hline BMI & & & & \\
$18.5-27.9$ & Reference & & & \\
$\geqq 28$ & -0.085 & 0.918 & $0.348-2.421$ & 0.863 \\
\hline$<18.5$ & 1.526 & 4.601 & $1.514-13.982$ & 0.007 \\
\hline LVEF & 0.939 & 2.558 & $1.620-4.04$ & $<0.001$ \\
\hline GLS & 1.299 & 3.307 & $2.062-5.304$ & $<0.001$ \\
\hline
\end{tabular}

BMI body mass index; LVEF left ventricular ejection fraction; GLS global longitudinal strain 


\section{Discriminative ability and performances of LCOS risk nomogram}

The C-index was calculated to evaluate the discriminative ability of the model, and the resulting value was $0.826(95 \% \mathrm{Cl}, 0.779-0.873)$ in the development group and $0.783(95 \% \mathrm{Cl}, 0.705-0.861)$ in the validation group. Bootstrap was used to verify the over-fitting of the estimation model. The C-index was 0.812 and 0.766 after calibration for development and validation group, respectively. The ROC curves were plotted to evaluate the discrimination ability and were shown in Fig 4 . These results suggest the nomogram prediction model has an excellent discrimination. Furthermore, we conducted a calibration plot for our prediction model, and a favorable agreement was shown between the actual and estimated probability of LCOS in the development group and validation group (Fig. 5).

\section{Presentation of a nomogram and clinical use}

The decision curve analysis for the LCOS nomogram is presented in Fig. 6. We did DCA on our prediction model to assess the net benefit that patients could receive. As the decision curve indicates, this nomogram achieved the most clinical utility to predict LCOS when the threshold probability for a patient is within a range from 0.03 to 0.66 .

\section{Discussion}

The present study is the first time to establish a nomogram for LCOS based on the perioperative risk factors after HVR including BMI, LVEF and GLS. The AUCs for the development and validation groups both exceeded 0.75 , indicating the prediction model has statistically significant discriminatory powers. According to the risk factors of LCOS shown in the nomogram model, might be beneficial for predicting outcomes of cardiac function and developing regimens to prevent LCOS.

According to our nomogram (Fig. 2), we can identify the point corresponding to the value of each predictor, and then sum these points together. The total point is associated with a probability that the patient will develop LCOS. For example, a patient underwent MVR with a BMI of 20, LVFE 55\%, and GLS 15. Totaling the points for this patient was 137.5 points in risk of LCOS of nomogram. This results in estimated LCOS rates of $41 \%$ according to the nomogram.

As the most common and the most serious complication after cardiovascular surgery, LCOS affects the prognosis of the patients and increases the rate of death seriously [4]. In our study, the mortality rate $(15.2 \%$ vs. $1.2 \%, p<0.001)$, ICU stay $(4(3-5.75)$ vs. $2(2-4), p<0.001)$ and the hospital stay $(29$ (26-33.75) vs. $24(22-28), p<0.001)$ were significantly higher in LCOS patients compared to those without LCOS. The widely accepted characteristics of LCOS included decreased heart pump function and the accompanying tissue hypoperfusion and hypoxia. However, there is no stringent diagnostic criterion of a low cardiac output state. In our study, we used a generally accepted clinical definition of low cardiac output syndrome, including a systolic blood pressure of less than $90 \mathrm{mmHg}$ for at least 30 minutes or postoperative need for IABP and/or prolonged requirement for inotropic support. Low cardiac output syndrome occurs in about $18.4 \%$ of patients in our study. This incidence of LCOS is greater than the 
prevalence of previous reports on isolated mitral and aortic valve surgery (3.9 and 7\%, respectively) $[3,6]$ but lower than the more recent reports $(21.5 \%$ and $41 \%$, respectively) $[7,8]$. The differences in the prevalence of LCOS in our population can be explained by the different type of cardiac disease, demographic characteristics and definition selected.

As World Health Organization BMI classification was not very suitable for Asian, we adopted a China classification and divided patients into the following 3 groups: low weight $\leqq 18.5$, normal-over weight $18.5-27.9$ and obese $\geqq 28$ [16]. BMI $<18.5$ is one option requires defining malnutrition [17]. Malnutrition is associated with a 2-fold increase in the probability of postoperative inotropic support and independently predicts adverse clinical outcomes [14]. Furthermore, low-weight may also be a manifestation of other associated comorbidities, such as cachexia, frailty or severe chronic diseases [18]. In our study, consistent with previous research [19], we found that the risk for LCOS was higher in low weight population. The low-weight patients were older, weaker, more severe impairment of left ventricular ejection, and more complex surgery. We consider these could be the main reason why the LCOS incidence was higher in low-weight patients than that in normal weight. The relationship between obesity and adverse outcomes include LCOS after cardiac surgery were conflicting among previous reports. For instance, some studies concluded that obesity was significantly associated with increased risk of LCOS and other postoperative morbidities [20-22]. Whereas others reported that obesity was a protective factor for postoperative complications [23-25]. Furthermore, multiple studies have suggested a U-shaped relationship between BMI and mortality[26]. However, in the present study, there was no significant correlation between obesity and LCOS risk after HVR.

Left ventricular ejection fraction (LVEF) based on visual analysis of two-dimensional (2D) images or Simpson biplane method is the most widely used parameters to assess the left ventricular systolic function [27]. A decreased LVEF is an independent risk factor for both LCOS and mortality after cardiac surgery, and have been included in risk models such as EUROSCORE [28]. Impaired left ventricular function (LVEF $<50 \%$ ) as an independent significant risk factor for LCOS has been described in some studies $[29,30]$. Consistent with previous research, impaired LVEF was an independent risk factor for LCOS after HVR in our study. However, influence of LVEF is less significant in patients underwent heart valve surgery compared with the CABG $[31,32]$. As ejection fraction is highly dependent on loading conditions, heart valve disease such as MR or AR can mask underlying LV dysfunction. Although the measurement of the LV function is normal and without obvious clinical symptoms, LV function is impaired $[6,7,33]$. In addition, this method heavily dependent on image quality and operator experience, and with significant inter-observer variability [34,35].

Myocardial deformation imaging (Strain imaging) is an echocardiographic technique to directly quantify the extent of myocardial contractility. The most widely used parameter to detect LV systolic function is GLS [27]. Advantages of using GLS to assess LV systolic function compared to LVEF include better reproducibility, ability to identify more subtle alterations in the contractility of the left ventricle, nonreliance on geometric assumptions, and lack of influence by tethering effects [35]. Recent studies suggest that the GLS measured during preoperative transthoracic echocardiogram (TTE) was shown to predict 
early postoperative mortality and the need for a postoperative inotropic support in patients with LVEF > $50 \%$ undergoing HVR [36]. Although the incremental value of GLS over other established risk factors for postoperative LCOS is limited in a study which included all kinds of on-pump cardiac surgery [37], it has been considered as the most significant parameter for LCOS risk in patients with AS without severely depressed LVEF [7]. Consistent with previous studies, the results in our study indicated that GLS was an independent risk factor for LCOS after HVR. If the GLS $\geqq-16$, the total score of the monogram is more than 100 , suggesting that GLS value can increase the LCOS risk of patients by $50 \%(100 / 200)$. In this study, we only included patients without severely depressed LVEF $($ LVEF $<40 \%)$, this result reflected indirectly a higher degree of LV subclinical dysfunction in our patients.

The study has several limitations. First, this is a retrospective cohort with limited cases, which cannot avoid selection bias; thus, prospective study with a larger sample size is needed to further confirm our conclusion. Second, our study is from a single center; although we validated the model by patient samples from different periods, it will be better to validate the model with data from another institution. Finally, we concerned only on patients undergoing valve replacement surgery without severely depressed LVEF and the data on other kinds of on-pump cardiac surgery are lacking; that makes the nomogram validated in this study may not be suitable for all patients. In spite of these drawbacks, this study firstly builds a nomogram of predicting LCOS in HVR patients.

\section{Conclusion}

In this study, we developed and validated an individualized nomogram prediction model for LCOS in patients undergoing HVR. Although we can accurately predict the risk of LCOS by this prediction model, there remains much work to do to refine the nomogram and to evaluate its clinical practicality.

\section{Abbreviations}

LCOS Low cardiac output syndrome; HVR heart valve replacement; LASSO least absolute shrinkage and selection operator; DCA decision curve analysis; TMVR transcatheter mitral valve replacement; TAVR transcathether aortic valve replacement; aCMQ automated cardiac motion quantification; CPB cardiopulmonary bypass; PCWP pulmonary capillary wedge pressure; IABP intra-aortic balloon pump; ROC receiver operator characteristic; BMI body mass index; NYHA New York Heart Association; AF atrial fibrillation; CHF congestive heart failure; SCR serum creatinine; BUN blood urea nitrogen; AV aortic valve; MV mitral valve; CBPT cardiopulmonary bypass time; ACCT aortic cross-clamp time; LVEF left ventricular ejection fraction; GLS global longitudinal strain; LVEDD left ventricular end diastolic diameter; LVESD left ventricular end systolic diameter; IVST interventricular septum thickness; LVPWT left ventricular posterior wall thickness; LAD left atrial diameter

\section{Declarations}

Acknowledgments 
Not applicable.

\section{Funding}

This study was funded by the Deyang Science and Technology Programs (2018SZS082) and (2018SZS081)

\section{Availability of data and materials}

All data generated or analyzed during the current study are available from the corresponding author on reasonable request.

\section{Authors' contributions}

QQ was a major contributor in writing the manuscript. XW, HZ, ZW analyzed and interpreted data. XS and JY drafted tables. PC contributed to conception and design. All authors read and approved the final manuscript.

\section{Ethics approval and consent to participate}

All study methods were approved by the Ethics Committee of People's Hospital of DeyangCity.

\section{Consent for publication}

All patients gave written formal consent to participate.

\section{Competing interests}

The authors declare that they have no competing interests.

\section{References}

1. Domenech B, Pomar JL, Prat-González S, Vidal B, López-Soto A, Castella M, et al. Valvular Heart Disease Epidemics. The Journal of heart valve disease. 2016;25(1):1-7.

2. Vahanian A, Alfieri O, Andreotti F, Antunes MJ, Barón-Esquivias G, Baumgartner H, et al. Guidelines on the management of valvular heart disease (version 2012): the Joint Task Force on the Management of Valvular Heart Disease of the European Society of Cardiology (ESC) and the European Association for Cardio-Thoracic Surgery (EACTS). European journal of cardio-thoracic surgery : official journal of the European Association for Cardio-thoracic Surgery. 2012;42(4):S1-44. doi: 10.1093/ejcts/ezs455.

3. Maganti MD, Rao V, Borger MA, Ivanov J, David TE. Predictors of low cardiac output syndrome after isolated aortic valve surgery. Circulation. 2005;112(9 Suppl):1448-52. doi:

10.1161/circulationaha.104.526087.

4. Lomivorotov VV, Efremov SM, Kirov MY, Fominskiy EV, Karaskov AM. Low-Cardiac-Output Syndrome After Cardiac Surgery. Journal of cardiothoracic and vascular anesthesia. 2017;31(1):291-308. doi: 
10.1053/j.jvca.2016.05.029.

5. Duncan AE, Kartashov A, Robinson SB, Randall D, Zhang K, Luber J, et al. Risk factors, resource use, and cost of postoperative low cardiac output syndrome. The Journal of thoracic and cardiovascular surgery. 2020. doi: 10.1016/j.jtcvs.2020.06.125.

6. Maganti M, Badiwala M, Sheikh A, Scully H, Feindel C, David TE, et al. Predictors of low cardiac output syndrome after isolated mitral valve surgery. The Journal of thoracic and cardiovascular surgery. 2010;140(4):790-6. doi: 10.1016/j.jtcvs.2009.11.022.

7. Balderas-Muñoz K, Rodríguez-Zanella H, Fritche-Salazar JF, Ávila-Vanzzini N, Juárez Orozco LE, Arias-Godínez JA, et al. Improving risk assessment for post-surgical low cardiac output syndrome in patients without severely reduced ejection fraction undergoing open aortic valve replacement. The role of global longitudinal strain and right ventricular free wall strain. The international journal of cardiovascular imaging. 2017;33(10):1483-9. doi: 10.1007/s10554-017-1139-6.

8. Yijun. L, Sheng. D, Rongsheng. X. Risk factors for low cardiac discharge syndrome in elderly patients after cardiac valve replacement. Guangxi Medical Journal. 2014;36(6):770-2.

9. Collier P, Phelan D, Klein A. A Test in Context: Myocardial Strain Measured by Speckle-Tracking Echocardiography. Journal of the American College of Cardiology. 2017;69(8):1043-56. doi: 10.1016/j.jacc.2016.12.012.

10. Dahl JS, Videbæk L, Poulsen MK, Rudbæk TR, Pellikka PA, Møller JE. Global strain in severe aortic valve stenosis: relation to clinical outcome after aortic valve replacement. Circulation Cardiovascular imaging. 2012;5(5):613-20. doi: 10.1161/circimaging.112.973834.

11. Kearney LG, Lu K, Ord M, Patel SK, Profitis K, Matalanis G, et al. Global longitudinal strain is a strong independent predictor of all-cause mortality in patients with aortic stenosis. European heart journal cardiovascular Imaging. 2012;13(10):827-33. doi: 10.1093/ehjci/jes115.

12. Mentias A, Alashi A, Naji P, Gillinov AM, Rodriguez LL, Mihaljevic T, et al. Exercise capacity in asymptomatic patients with significant primary mitral regurgitation: independent effect of global longitudinal left ventricular strain. Cardiovascular diagnosis and therapy. 2018;8(4):460-8. doi: 10.21037/cdt.2018.05.03.

13. Kim HM, Cho GY, Hwang IC, Choi HM, Park JB, Yoon YE, et al. Myocardial Strain in Prediction of Outcomes After Surgery for Severe Mitral Regurgitation. JACC Cardiovascular imaging. 2018;11(9):1235-44. doi: 10.1016/j.jcmg.2018.03.016.

14. Lomivorotov VV, Efremov SM, Boboshko VA, Nikolaev DA, Vedernikov PE, Lomivorotov VN, et al. Evaluation of nutritional screening tools for patients scheduled for cardiac surgery. Nutrition (Burbank, Los Angeles County, Calif). 2013;29(2):436-42. doi: 10.1016/j.nut.2012.08.006.

15. Sauerbrei W, Royston P, Binder H. Selection of important variables and determination of functional form for continuous predictors in multivariable model building. Statistics in medicine. 2007;26(30):5512-28. doi: 10.1002/sim.3148.

16. Appropriate body-mass index for Asian populations and its implications for policy and intervention strategies. Lancet (London, England). 2004;363(9403):157-63. doi: 10.1016/s0140-6736(03)15268- 
3.

17. Cederholm T, Bosaeus I, Barazzoni R, Bauer J, Van Gossum A, Klek S, et al. Diagnostic criteria for malnutrition - An ESPEN Consensus Statement. Clinical nutrition (Edinburgh, Scotland). 2015;34(3):335-40. doi: 10.1016/j.clnu.2015.03.001.

18. Preston SH, Stokes A. Obesity paradox: conditioning on disease enhances biases in estimating the mortality risks of obesity. Epidemiology (Cambridge, Mass). 2014;25(3):454-61. doi: 10.1097/ede.0000000000000075.

19. Burgos LM, Gil Ramírez A, Seoane L, Espinoza J, Furmento JF, Costabel JP, et al. Is the Obesity Paradox in Cardiac Surgery Really a Myth? Effect of Body Mass Index on Early and Late Clinical Outcomes. Journal of cardiothoracic and vascular anesthesia. 2021;35(2):492-8. doi: 10.1053/j.jvca.2020.03.051.

20. Lio A, Bovio E, Nicolò F, Saitto G, Scafuri A, Bassano C, et al. Influence of Body Mass Index on Outcomes of Patients Undergoing Surgery for Acute Aortic Dissection: A Propensity-Matched Analysis. Texas Heart Institute journal. 2019;46(1):7-13. doi: 10.14503/thij-17-6365.

21. Ho KM, Bertenshaw C, Same S, Schneider M, Williams KA, Godsell T, et al. Differential associations between body mass index and outcomes after elective adult cardiac surgery: a linked data cohort study. Anaesthesia and intensive care. 2013;41(5):573-83. doi: 10.1177/0310057x1304100502.

22. Prasad US, Walker WS, Sang CT, Campanella C, Cameron EW. Influence of obesity on the early and long term results of surgery for coronary artery disease. European journal of cardio-thoracic surgery : official journal of the European Association for Cardio-thoracic Surgery. 1991;5(2):67-72; discussion -3. doi: 10.1016/1010-7940(91)90003-3.

23. Mariscalco G, Wozniak MJ, Dawson AG, Serraino GF, Porter R, Nath M, et al. Body Mass Index and Mortality Among Adults Undergoing Cardiac Surgery: A Nationwide Study With a Systematic Review and Meta-Analysis. Circulation. 2017;135(9):850-63. doi: 10.1161/circulationaha.116.022840.

24. Stamou SC, Nussbaum M, Stiegel RM, Reames MK, Skipper ER, Robicsek F, et al. Effect of body mass index on outcomes after cardiac surgery: is there an obesity paradox? The Annals of thoracic surgery. 2011;91(1):42-7. doi: 10.1016/j.athoracsur.2010.08.047.

25. Hartrumpf M, Kuehnel RU, Albes JM. The obesity paradox is still there: a risk analysis of over 15000 cardiosurgical patients based on body mass index. Interactive cardiovascular and thoracic surgery. 2017;25(1):18-24. doi: 10.1093/icvts/ivx058.

26. Burns DJP, Rapetto F, Angelini GD, Benedetto U, Caputo M, Ciulli F, et al. Body mass index and early outcomes following mitral valve surgery for degenerative disease. The Journal of thoracic and cardiovascular surgery. 2019. doi: 10.1016/j.jtcvs.2019.10.193.

27. Lang RM, Badano LP, Mor-Avi V, Afilalo J, Armstrong A, Ernande L, et al. Recommendations for cardiac chamber quantification by echocardiography in adults: an update from the American Society of Echocardiography and the European Association of Cardiovascular Imaging. European heart journal cardiovascular Imaging. 2015;16(3):233-70. doi: 10.1093/ehjci/jev014. 
28. Nashef SA, Roques F, Michel P, Gauducheau E, Lemeshow S, Salamon R. European system for cardiac operative risk evaluation (EuroSCORE). European journal of cardio-thoracic surgery : official journal of the European Association for Cardio-thoracic Surgery. 1999;16(1):9-13. doi: 10.1016/s1010-7940(99)00134-7.

29. Ding W, Ji Q, Shi Y, Ma R. Predictors of low cardiac output syndrome after isolated coronary artery bypass grafting. International heart journal. 2015;56(2):144-9. doi: 10.1536/ihj.14-231.

30. Sá MP, Nogueira JR, Ferraz PE, Figueiredo OJ, Cavalcante WC, Cavalcante TC, et al. Risk factors for low cardiac output syndrome after coronary artery bypass grafting surgery. Revista brasileira de cirurgia cardiovascular : orgao oficial da Sociedade Brasileira de Cirurgia Cardiovascular. 2012;27(2):217-23. doi: 10.5935/1678-9741.20120037.

31. Rao V, Christakis GT, Weisel RD, Buth KJ, Ikonomidis JS, Shirai T, et al. Changing pattern of valve surgery. Circulation. 1996;94(9 Suppl):li113-20.

32. Weisel RD, Burns RJ, Baird RJ, Hilton JD, Ivanov J, Mickle DA, et al. Optimal postoperative volume loading. The Journal of thoracic and cardiovascular surgery. 1983;85(4):552-63.

33. Weidemann F, Jamal F, Sutherland GR, Claus P, Kowalski M, Hatle L, et al. Myocardial function defined by strain rate and strain during alterations in inotropic states and heart rate. American journal of physiology Heart and circulatory physiology. 2002;283(2):H792-9. doi:

10.1152/ajpheart.00025.2002.

34. Kalogeropoulos AP, Georgiopoulou VV, Gheorghiade M, Butler J. Echocardiographic evaluation of left ventricular structure and function: new modalities and potential applications in clinical trials. Journal of cardiac failure. 2012;18(2):159-72. doi: 10.1016/j.cardfail.2011.10.019.

35. Velagapudi VM, Pidikiti R, Tighe DA. Is Left Ventricular Global Longitudinal Strain by TwoDimensional Speckle Tracking Echocardiography in Sepsis Cardiomyopathy Ready for Prime Time Use in the ICU? Healthcare (Basel, Switzerland). 2019;7(1). doi: 10.3390/healthcare7010005.

36. Ternacle J, Berry M, Alonso E, Kloeckner M, Couetil JP, Randé JL, et al. Incremental value of global longitudinal strain for predicting early outcome after cardiac surgery. European heart journal cardiovascular Imaging. 2013;14(1):77-84. doi: 10.1093/ehjci/jes156.

37. Amabili P, Benbouchta S, Roediger L, Senard M, Hubert MB, Donneau AF, et al. Low Cardiac Output Syndrome After Adult Cardiac Surgery: Predictive Value of Peak Systolic Global Longitudinal Strain. Anesthesia and analgesia. 2018;126(5):1476-83. doi: 10.1213/ane.0000000000002605.

\section{Figures}




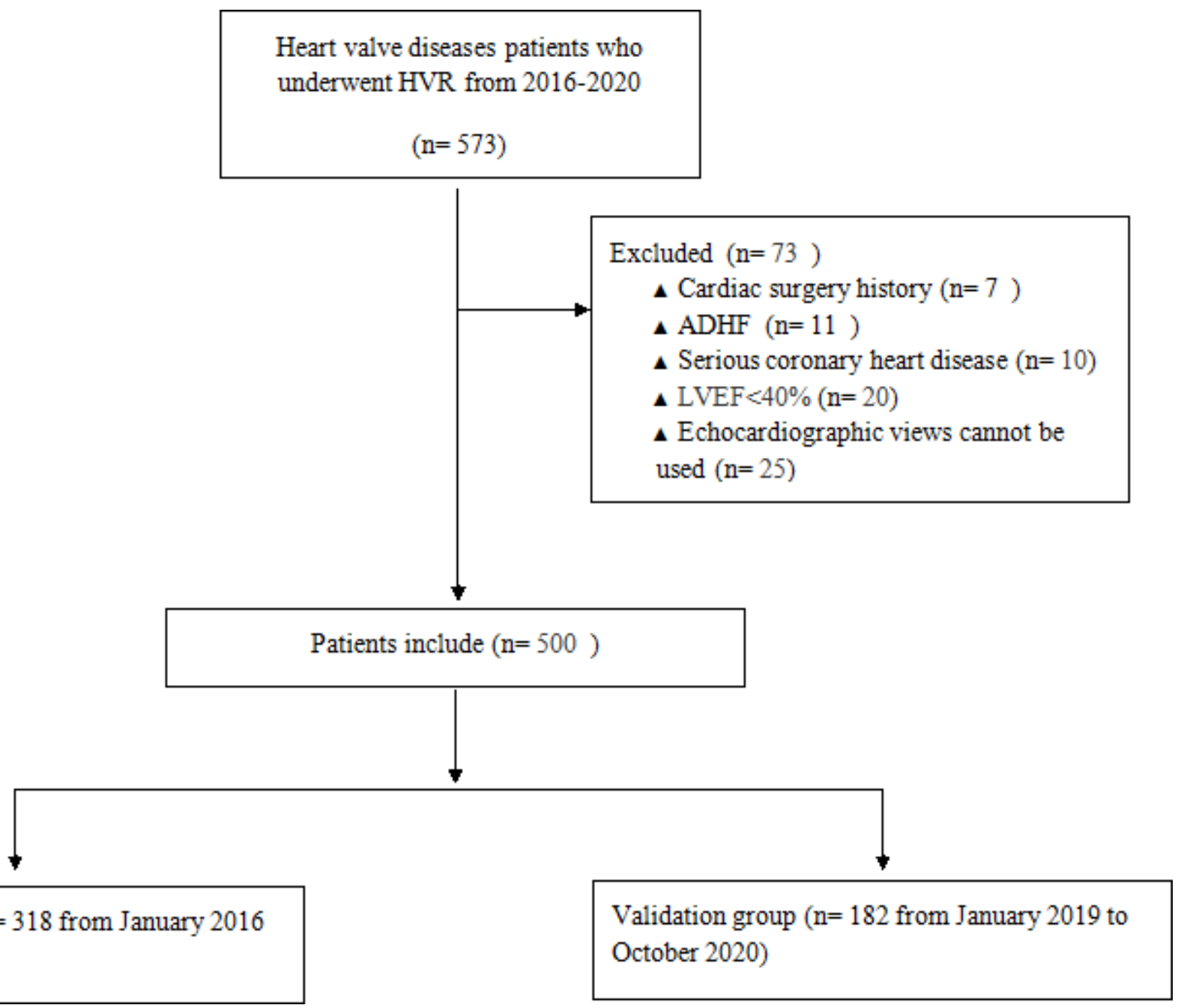

\section{Figure 1}

The flow chart 
$\begin{array}{lllllllllllllllllllllllllll}\mathrm{A} & 10 & 10 & 10 & 10 & 10 & 9 & 8 & 8 & 8 & 6 & 3 & 2 & 2 & 2 & 2 & 2 & 1 & 0 & 0 & 0 & 0 & 0 & 0 & 0 & 0 & 0\end{array}$
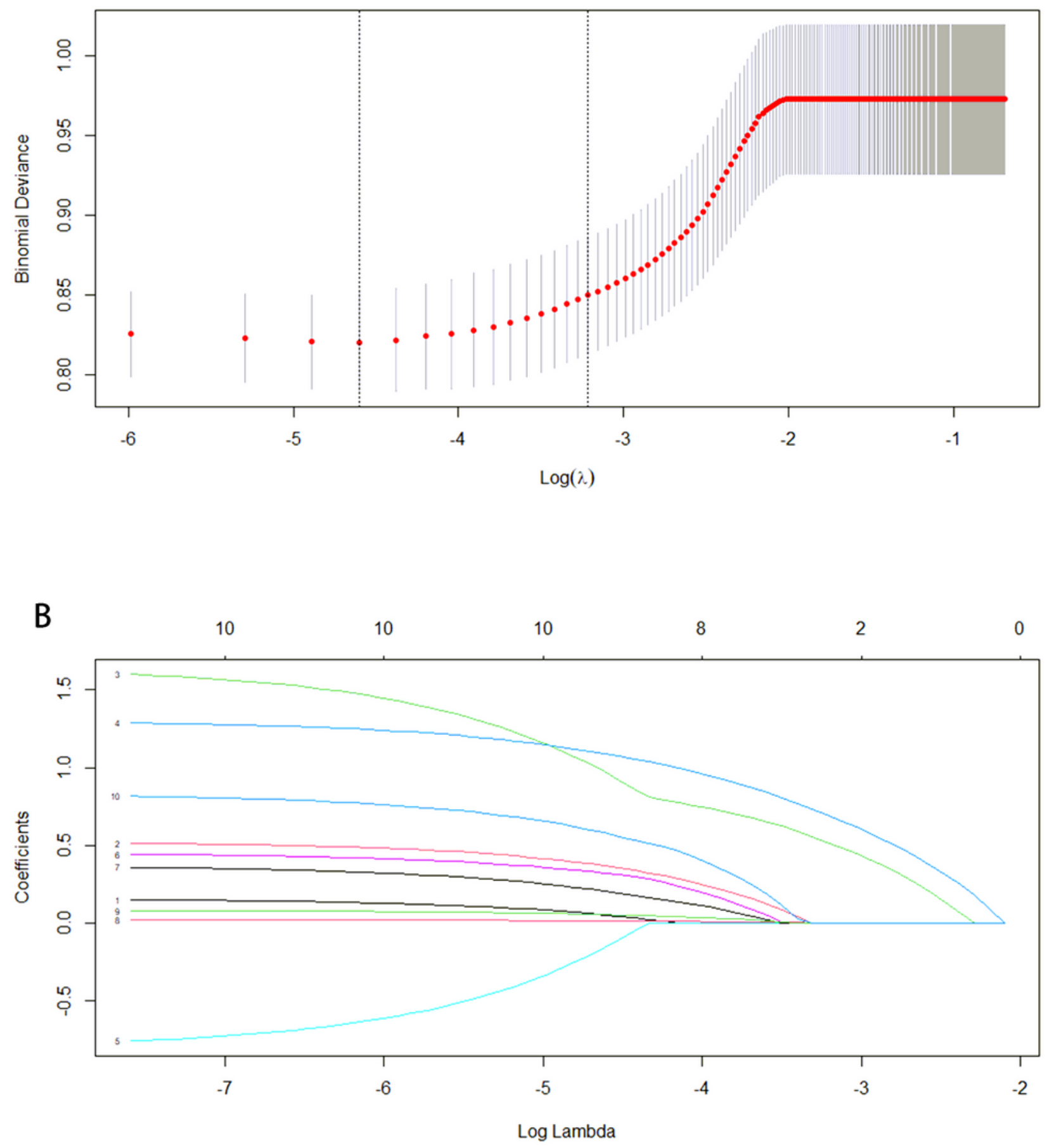

Figure 2

Predictor selection by the LASSO. A optimal penalization coefficient lambda $(\lambda)$ selection by LASSO used fourfold cross-validation and the minimum criterion. B LASSO coefficient profiles of the 10 variables plotted against the log (lambda) sequence. The minimum criterion and the 1 standard error of the minimum criteria are used to draw the dotted vertical line at the optimum value. 
Points

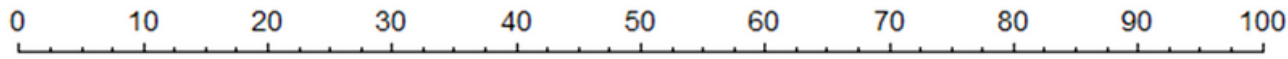

LW

LVEF

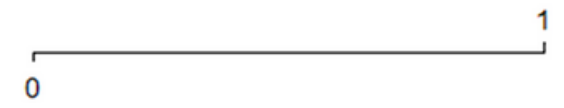

1

$50-60$

LVEF

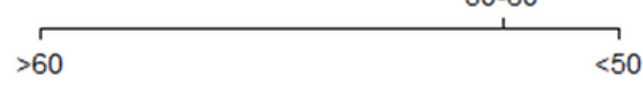

GLS

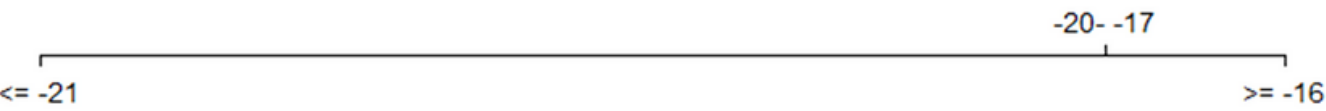

Total Points

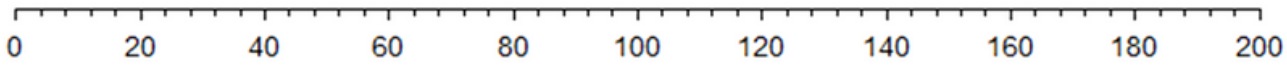

LCOS rate

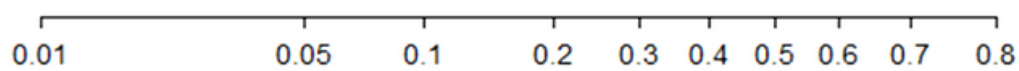

\section{Figure 3}

A nomogram to predict the the probability of LCOS after HVR. A nomogram for LCOS was developed and integrated with BMI, LVEF, and GLS. Abbreviations: LCOS low cardiac output syndrome; HVR heart valve replacement; BMI body mass index; LW low weight; LVEF left ventricular ejection fraction; GLS global longitudinal strain.

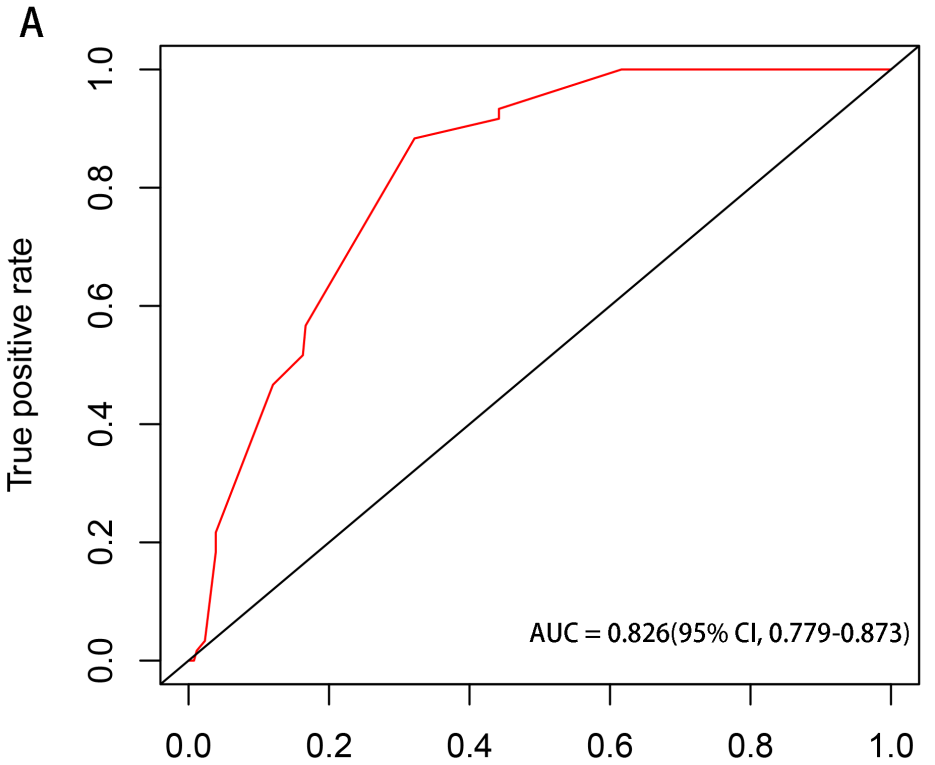

False positive rate

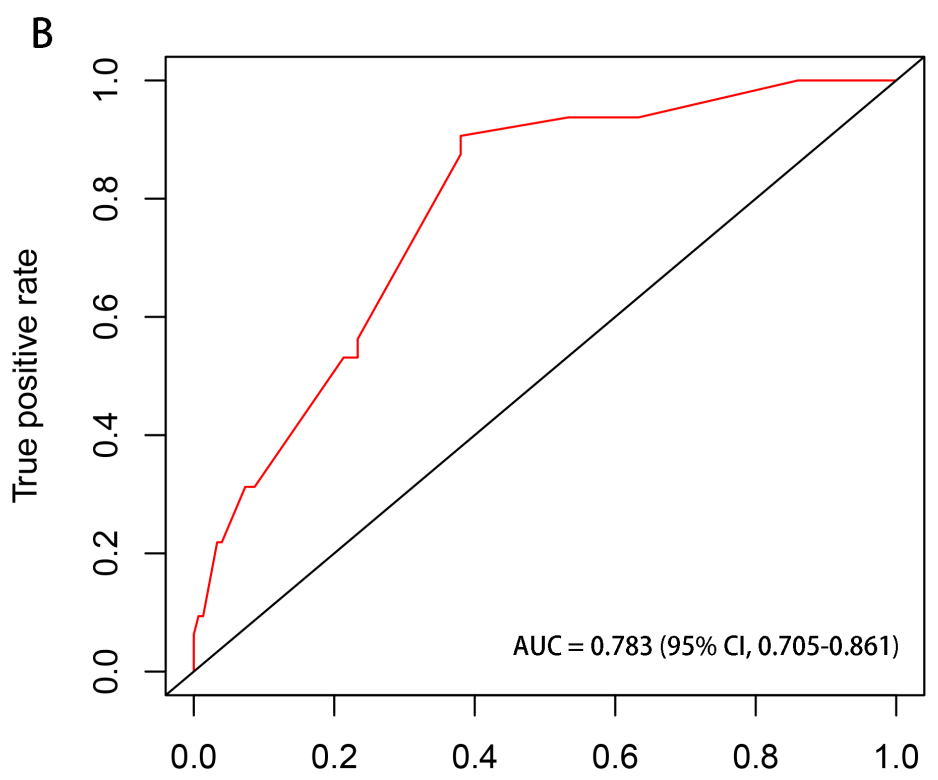

False positive rate

Figure 4 
ROC analysis of the nomogram for LOCS. ROC curve for discrimination in the development group and validation group. The AUCs of the nomogram were respectively 0.826 and 0.783 , demonstrating very good prediction performance. Abbreviations: ROC receiver operating characteristic; AUC area under curve

\section{A}

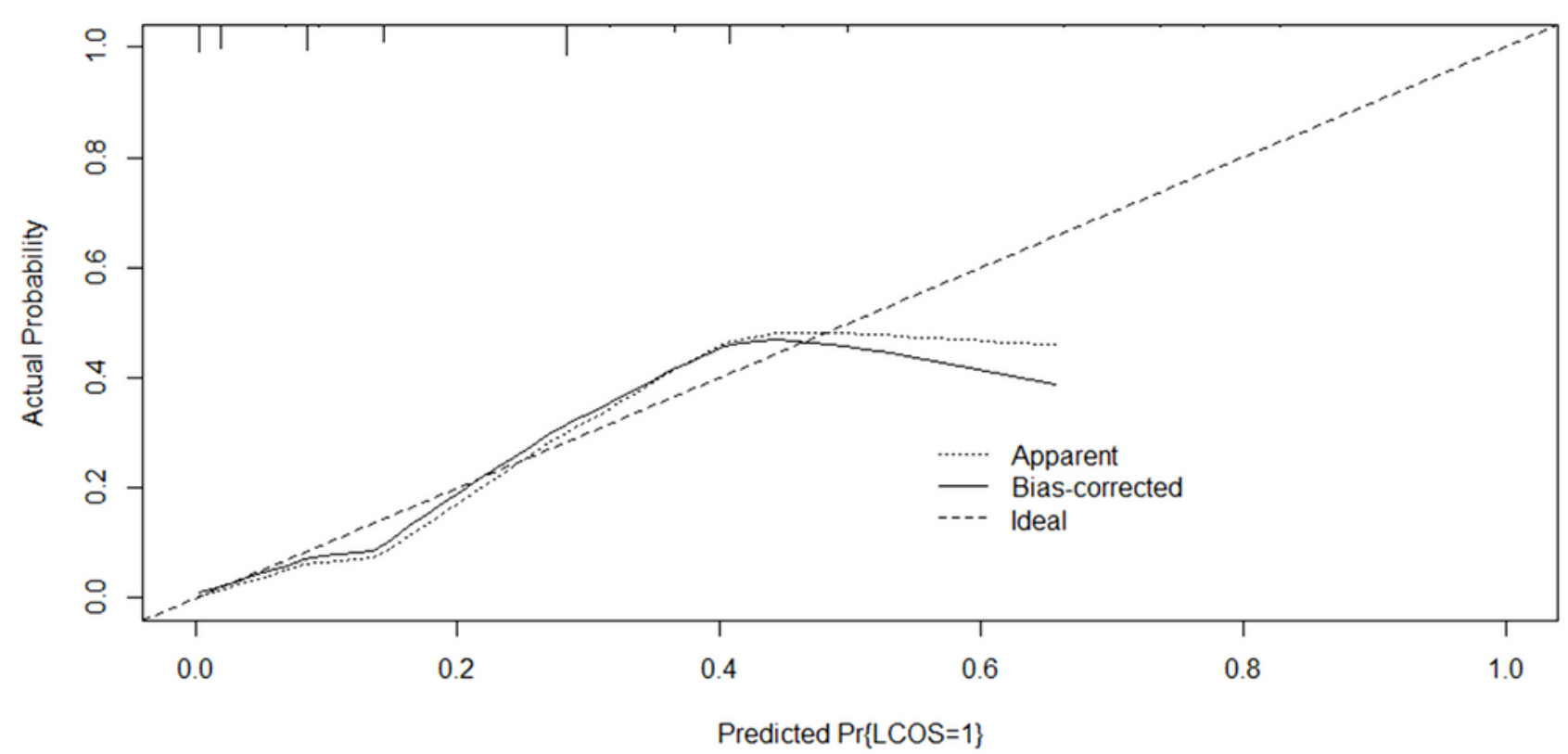

$B=1000$ repetitions, boot

Mean absolute error $=0.028 n=318$

B

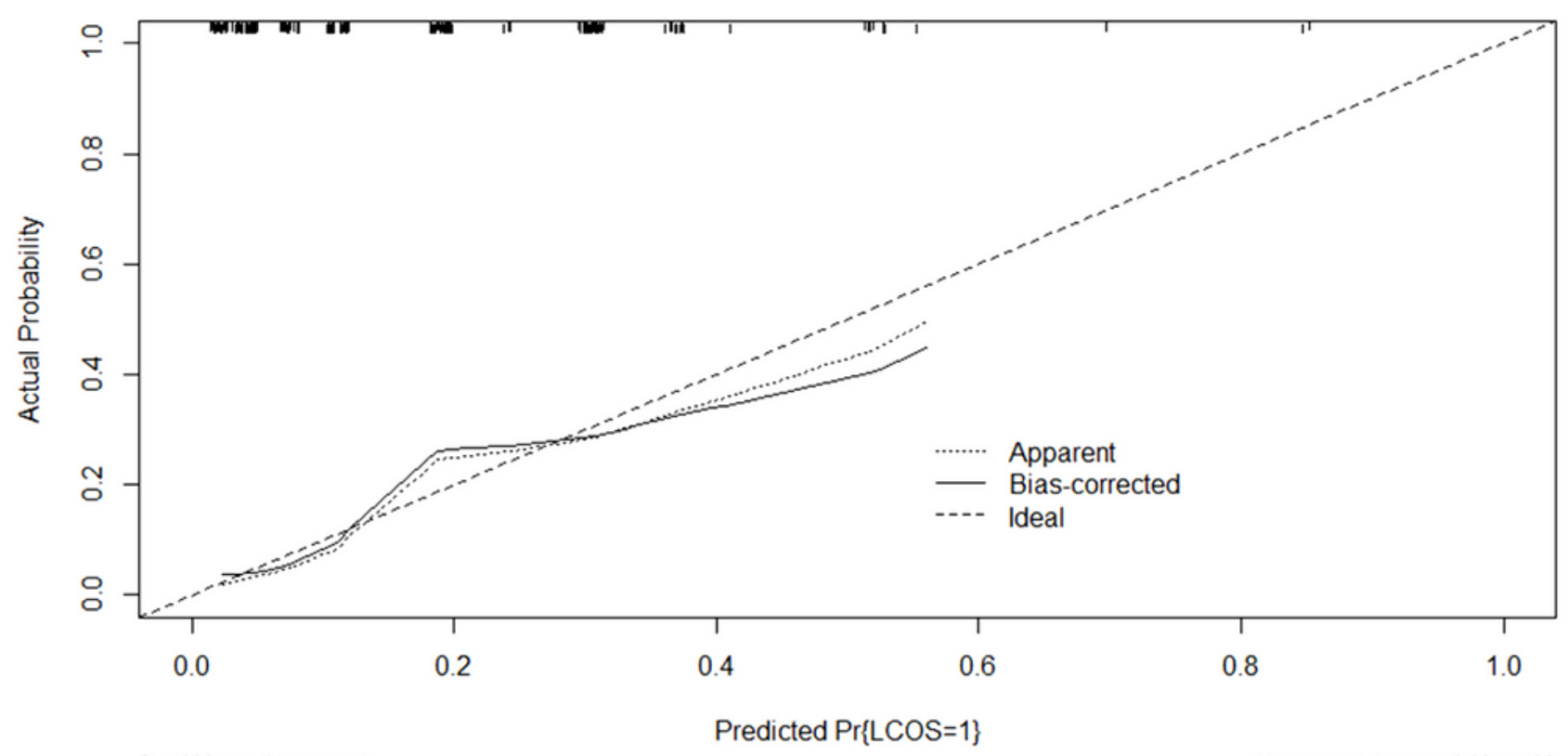

$B=1000$ repetitions, boot

Mean absolute error $=0.03 n=182$

Figure 5

Calibration curves of the LCOS prediction nomogram. The predicted LCOS risk is shown on the X-axis. The actual diagnosed LCOS is shown on the Y-axis. (A) Development group; (B) Validation group. 


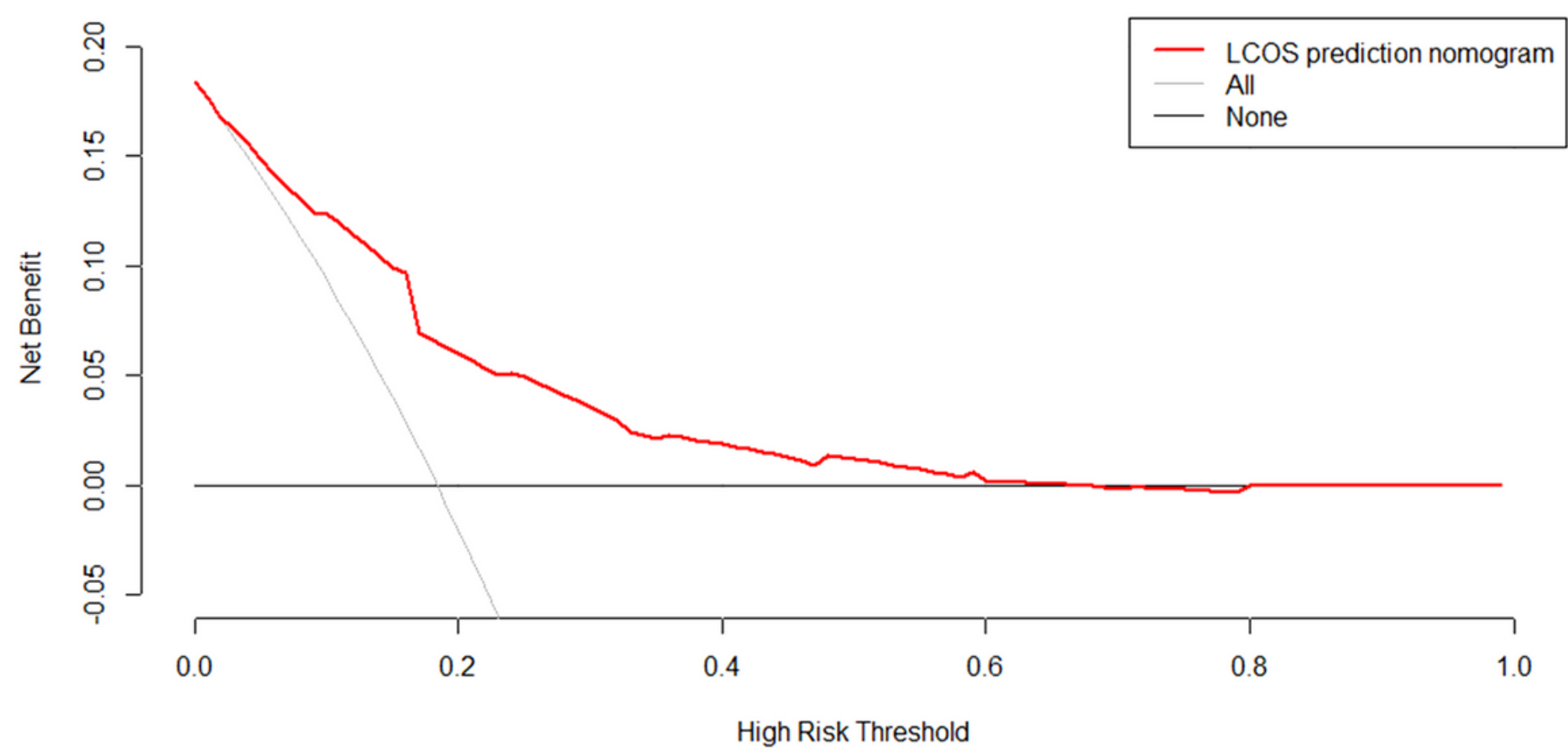

\section{Figure 6}

Decision curve analysis (DCA) for the LCOS prediction nomogram. The red line represents the LCOS prediction nomogram. The thin solid line represents the hypothesis that all patients had LCOS. The thick solid line represents the hypothesis that no patients had LCOS. The DCA in the validation cohort demonstrated that if the threshold probability is between 0.03 and 0.66 , the use of the nomogram to predict LOCS is more beneficial than treating all or no patients. 